$$
\begin{aligned}
& \text { SAND. -79.1479C } \\
& \text { CONE-790726--7 }
\end{aligned}
$$

\title{
MASTER
}

\section{LATCH-UP CONTROL IN \\ CMOS INTEGRATED CIRCUITS}

A. OCHOA AND W. DAWES

SANDIA LABORATORIES

ALBUQUERQUE, NEW MEXICO

D. ESTREICH

HEWLETT PACKARD

SANTA ROSA, CALIFORNIA

(7) Sandia Laboratories 


\section{MASTER}

SECOND DRAFT

July 13,1979

Latch-up Control in cmos Integrated circuits

\section{ABSTRACT}

The potential for latch-up, a pnpn self-sustaining low impedance state, is inherent ir. standard bulk CMOS-integrated circuit structures. Under normal bias, the parasitic ecR is in its blocking state but, if subjected to a large voltage spike or if exposed to an ionizing environment, triggering may occur. This may result in device burn-out or loss of state. The problem has been extensively studjed for space and weapons applications. Prevention of latch-up has keen achieved in conservative design $(\sim 9 \mu \pi$ p-well depths) by the use of minority lifetirie control methods such as gold doping and neutron irradiation and by modifying the base transport factor with buried layers. The push toward VLSI densities wiII enhanze parasitic action sufficiently so that the problem will become of more universal concern. Th:s paper will survey latch-up control methods presently employed.for weapons and 
space applications on present ( $9 \mu \mathrm{m}$ p-well) CMOs and will indicate the extent of their applicability to vus designs.

\section{INTRODUCTION}

Latch-up in bulk, cMOs-integrated circuits occurs due to parasitic four layer pnpn paths. The transistors involved in such a path are identified in Figure ]. Under normal operation, the emitter-base junctions of both transistors conduct only leakage current and the structure is in its blocking state. If enough lateral current can be made to flow, the transistors become forward-biased and regeneration can be initiated. Once attained, functionality is lost and device destruction may follow--metallization or junction failule. The smaller structures required for higher packing densities will entance the pioblem. Bulk CMOS having an $\sim 9$ micron p-well depth (hereinaf ier referred to as standard CMOS) has been made free of the latch-up problem by the generic solution of reduction of minority lifetimie. The same techniques, however, will not by themselves be sufficient to guarantee workable, latch-up free cMos circuits in reduced design rule structures. This paper will review latch-up prevention in present standard CMOS, in particular, discuss a CMOS process on an epitaxial substrate that made a test device free of latch-up. A simple 
SECOND DRAFT, $7 / 13 / 79$

$-3-$

analysis is presented to explain the obtained protection. With the prevention methods in mind, we then present a process outline that should be latch-up free for the smaller structures.

\section{LATCH-UP CONTROL IN STANDAKD CMOS}

In Figure 1 we show a cross-section of the standard bulk cmos structure with the parasitic bipolar devices involved in the latch-up process identified. Also shown is a lumped, First-order equivalent model. Latch-up is possible only if (I) the transistors become biased into the forward active mode, (2) the transistor current gain product ( $\beta_{\text {npn }}{ }^{-\beta}$ pnp' exceeds a required minimum for regeneration to occur, and (3) the bias supply is capable of sourcing a current greater than the holding current. Prevention of any of these requirements will prevent latch-up.

At present, the principal methods used concern requirement two above. Since the current gains of the standard CMos structure are usually base tránsport limited, minority iffetime control proved to be a useful parameter to focus upon. Diffusing: gold at temperatures greater than $800^{\circ} \mathrm{C}$ to obtain concentrations of $10^{15} / \mathrm{cc}$ of gold was found to produce the desired results (1)--minority lifetimes less than 100 ns and $\beta_{\text {npn }} \equiv 3$. Fast neutron irradiation to a level of $30^{24} \mathrm{~N} / \mathrm{cm}^{2}$ 
of energies greater than . $1 \mathrm{Mev}(2,3)$ produced similar results. These methods were attractive because 1 ittle process modification was required and few undesirable additional. effects were introduced.

Current gain is also reduced by appropriate base concentration profiling. A buried layer morphology has been shown to reduce the base transport by use of an increased base Gummel number and a retaroing electric field (4). Except for the extra processing required for the buried layer itself, again the standard CMOS process was unaltered. A buried layer of $30 \Omega / 0$ and $\approx 13 \mu \mathrm{m}$ deep prior to the epitaxial layer deposition was used. The structure, shown in Figure 2, was immune to latch-up, a $\beta_{\text {npn }}$ of approximately 1 was obtained. $R_{S}$ and $R_{w}$ in Figure $l$ were substantially reduced by this process. The effect of these shunt resistors is itseif a potential control of latch-up as will now be discussed.

If the available power supply current is below the holding current of the pnpn path, evea if latch-up is initiated, it cannot be sustaired. Processing standard CMOS on F.pi-wafers ( $n$ on $n^{+}$) will reduce $R_{s}$ and raise the holding current as described in Eq. 1. 


$$
\begin{gathered}
I_{H}=\frac{I_{R s} \beta_{P}\left(\beta_{n}+1\right)+I_{R W} \beta_{n}\left(\beta_{P}+1\right)}{\beta_{n} \beta_{p}-I} \\
I_{R s}=\text { Current flowing through } R_{s} \\
I_{R W}=\text { Current flowing through } R_{w} \\
I_{H}=\text { Holding current }
\end{gathered}
$$

Since $I_{R S}$ increases substantially for an Epi structure, the holding current becomes quite large. For CMOS 4007 devices fabricated on Epi wafers, the holding current was made to exceed the level at which device destruction was obtained (junction failure). Caution is waranted here, however, as the structure is not immune to latch-up except under normal bias. If inputs are raised above $v_{D D^{\prime}}$ the conditions leading to Eq. 1 are not met, thus nullifying the results. Latch-up can occur, but the device was found to exit from latch upon returning to normal bias conditions, $v_{s 5} \leq V_{i} \leq v_{D D}$. A CMOs 4007 circuit processed upon an epitaxial substrate will latch-up if $V_{\text {op }}$ is left floating and an input is held positive with respect to $\mathrm{v}_{\mathrm{SS}}$. Making $\mathrm{v}_{\mathrm{DD}}$ equal to the most positive potential applied to the circuit keeps the device out of latch-up as shown in Figure 3b. In figure $3 a$ a standard device is shown. Note that tylng $v_{D D}$ to the most positive potential here raises the holding current. but latch-up is still otserved. The functional relationship expressed in Eg. 1 is shown in Figure 4. 
Addition of a parasitic emitter resistor indicates an additional Eavorable effect. The holding current now becomes

$$
I_{H}=\frac{\left(V_{\beta E}{ }_{p n p}+r_{e E} I_{D D} \beta P(\beta n+1)+\frac{\left(V_{\beta E}{ }_{n p n}+r_{e n} I_{D D} \beta_{n}\left(\beta_{p}+{ }^{\top}\right)\right.}{R_{S}+r_{e p}}\right.}{\beta n \beta p-1}
$$

with $r_{\text {ep }}=$ emitter series resistance of the pnp

$$
r_{\text {en }}=\text { emitter series resistance of the npn. }
$$

This effect is shown graphically in Figure 5.

Another useful effect of the epitaxial process concerns the current gain product requirement for latch-up. This is generally expressed as $\beta_{\mathrm{npn}} \boldsymbol{B}_{\mathrm{pnp}} \geqq$ I (6). If the -ipply current is Iimited, this requirement is too restrictive and should be reglaced by

$$
\beta_{n} \beta_{n} \geq \frac{I_{\text {max }}+I_{R W} \beta_{n}}{I_{\text {Dnax }}-I_{R w}-I_{R s} \frac{\beta_{n}+I}{\beta_{n}}}
$$

where $x_{\text {Dmax }}$ is the maximum ayailable supply current.

Gain products greater than one can be tolerated. The Epi CMos structure, for example, $\beta_{\text {npn }} \doteq 140$, and $B_{\text {pnp }} \doteq 25$, a gain product of 35 , did not latch. Note that an infinite available 
SECOND DRAFT', 7/13/79

source current will result in a $\beta$ product of 1 . Again, the emitter resistance will change Eq. 3 considerably for large values.

A particular latch-up path that is easily eliminated concerns the input protection circuitry. A p diffused region is generally used for series impedance and shunt diode clamping. Surrounding this region with a grounded p ring as shown in Figure, 6 : forms a pseudo collector that alters the path drastically. The $\beta_{\text {pnp }}$ reductions by factors of 140 have been obtained (7). Since these lateral devices normally exhibit gains of . 25 or less, the vertical npn must now have a gain in excess of 500 for the input circuitry to participate in latch-up.

\section{REDUCED DESIGN RULE CMOS}

To obtain increased packing densities, shallower p-wells and smaller surface separations must be used. Following general MOS scaling rules (8), a 2 micron gate scaled transistor with a $40 \mathrm{nM}$ gate oxide will have a density of 40,000 gates $/ \mathrm{cm}^{2}$. For such circuits, both parasitic transistors will have increased current gains and latch-up susceptability will increase. A test circuit with $4 \mu m$ channel length ano a 3.0 micron, $3500 \Omega / 0$ p-well was found to 
latch after $2 \times 10^{14} \mathrm{~N} / \mathrm{cm}^{2}$. Extrapolation of the gain fall-off with neutron irradiation, assuming a base transport dominated morel indicates that a dose of $3-5 \times 30^{1.5} \mathrm{~N} / \mathrm{cm}^{2}$ will be required for unity gain to be obtained in the vertical npn transistor. Such a fluence level is unacceptable as it ..... would require long exposures (approximately 36 hours at Sandia's Pulse Reactor) and be accompanied by total gamma dose in excess of $10^{5}$ Rads(si). Gold densities of $10^{16}, \mathrm{~cm}^{2}$ would be needed--enough to cause compensation problems.

A structure that will render shallow p-well cMos fiee from latch-up has evolved from these considerations. The p-well, 1.5-3 microns deep, will have a reduced surface concentration produced by use of a counter doped shallow As implant. A larger Gumel number is thus obtained while maintaining a low $10^{16} / \mathrm{cm}^{2}$ surface concentration. The substrate will be $n$ on $n^{+} E p i$ to reduce $R_{S}$ (increase $I_{H}$ ). To Eurther reduce the current gain of the vertical npn, an arsenic implant will be useō Eollowed by processing of less than $900^{\circ} \mathrm{C}$. This will result in a shallow poor emitter (9). Pseudo collectors about the input protection circuit could be included. 


\section{SUMMARY}

Latch-up in standard CMOS designs is controllable by use of minority lifetime reduction, gold diffusion or neutron irradiation. Other techniques that have shown control are epitaxial substrates, buried layer structures, and layout changes. The effects of smaller dimensions required for increased density bill be controllable only by combinations of these and, possibly, the addition of emitter efficiency reduction afforded by the use of incomplete annealed arsenic n-channel source/drains. 


\section{Eigures}

1a. Cross-section of a standard cMOs section showing a four layer path and the parasitic transistors.

Ib. Lumped equivalent model of pnpn parasitic.

2. Cross-section of the buried layer CMOs.

3a. Latch in steidard cMos. Lower holding current is for latch between an input and $\mathrm{v}_{\mathrm{SS}}$, the higher holding current is Eor $V_{i n}$ eparallel with $V_{D D}$ to $V_{S S}$.

3b. Latch in Epi-cMOs device. Latch-'? oscurs only if $V_{D D}$ is open. Putting $v_{D D}$ to the most positive potential prevents SCR action.

4. Holding current as a Eunction of Substrate Resistance, Rs.

5. Holding current as a Eunction of Emitter series Resistance, $\boldsymbol{I}$

6. Example of layout prevention of latch-up. The psuedo collector shunts any emitted current to ground preventing participation in latch-up. 
4
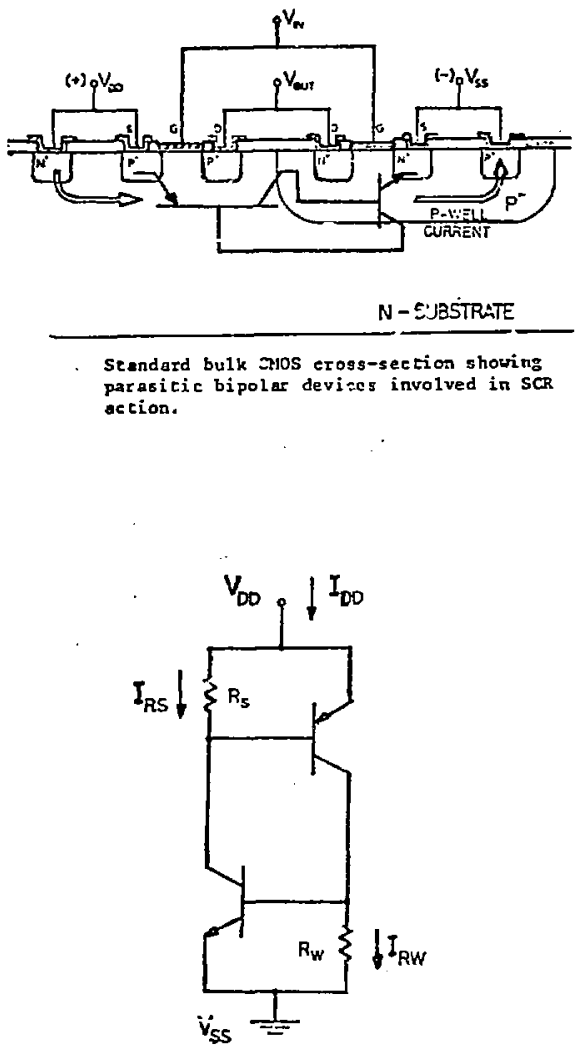

First-order equivalent circuit of the pnpn latch-up path in a bulk CMOS atructure.

11 

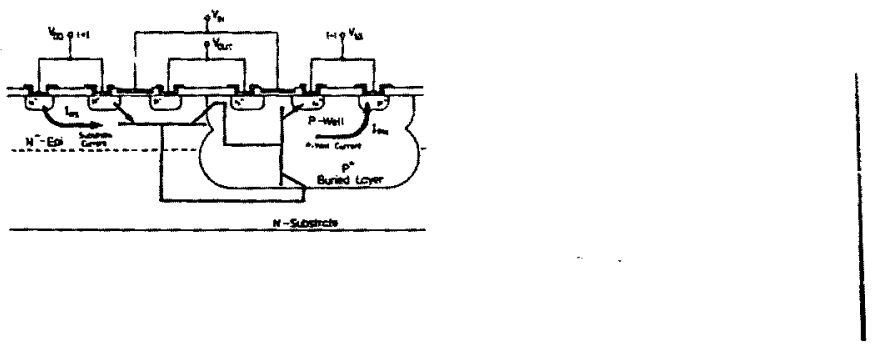

s 

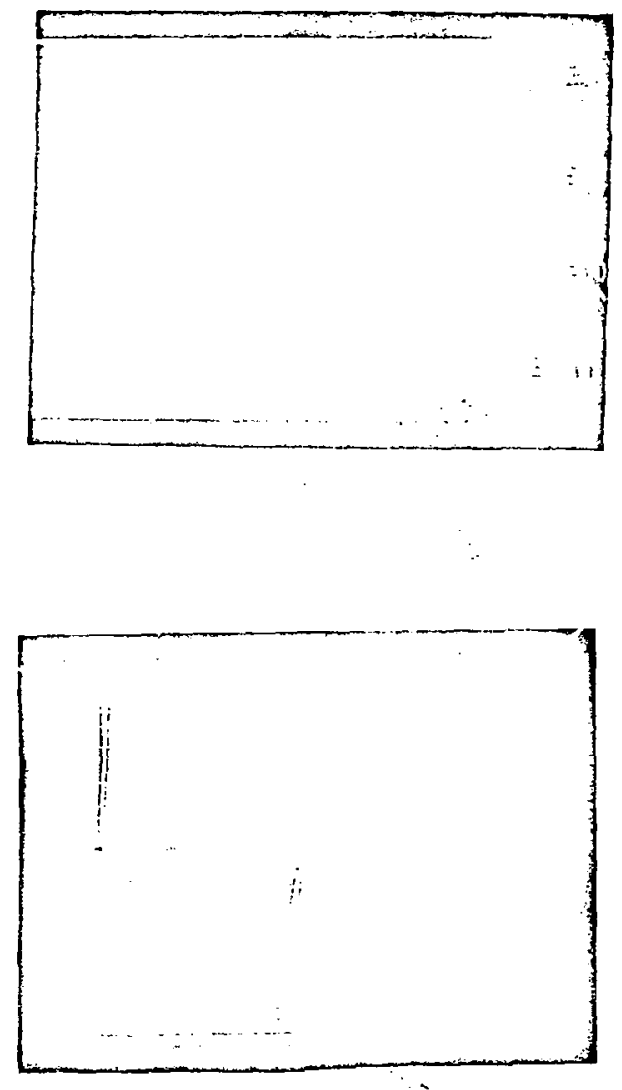

1 

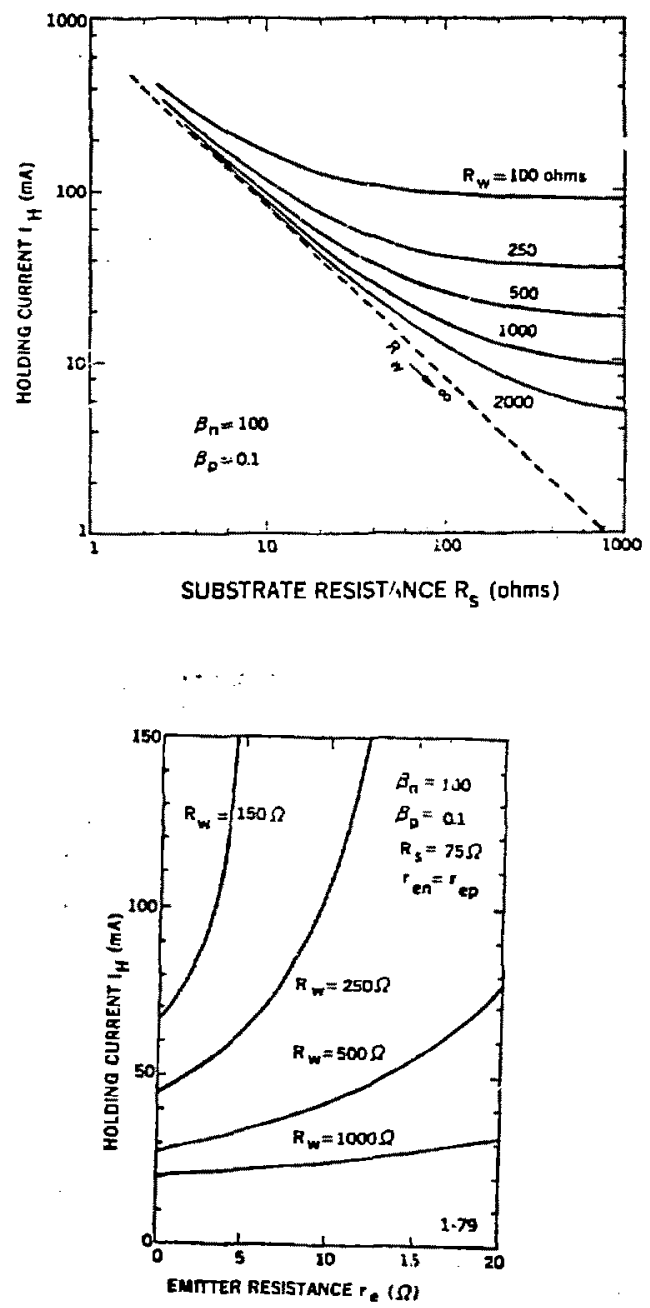


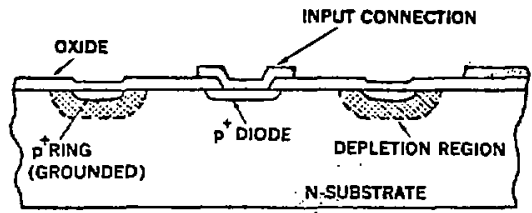

PSUEDO COLLECTOR STRUCTURE 
Refurences

1. W. R. Dawes and G. F. Derbenwick, "prevention of cmos Latch-Up by Gold Doping," IEEF Trans. On Nuclear Science, Vol. NS-23, No. 6, pp. 2027-2030, December 1976 .

2. J. R. Adams and R. J. Sokel, "Neutron Irradiation for Prevention of Latch-Up in MOS Integrated Circuits," IFFE Annual Conf. on Nuclear and Space Rad Effects, .1979.

3. C. E. Rarnes, et al, Latch-Up Prevention in CMos, Sandia Report SAND 76-0048, March 1976.

4. D. B. Estreich and A. Ochoa, Jr.. "An Analysis of Latch-Up Prevention in CMOS ICs Using an Epitaxial-Buried Layer Process," International Electron Device Mee'ing, Washington, D.C., 1978.

5. S. M. Sze, Prysics of Semiconauctor Devices, Wiley-Interscience, 3.969.

6. D. B. Estreich, Ph.D., Dissertation, Department of Electrfcal Engineering, stanford University, in progress. 
$-12-$

7. R. H. Dennard, et aI, "Design of Ion-Implanted MOSFETs with Very Small Physical Dimensions," IEEE J. uE Solid State Circuits, vol. sc-9 No. 5. Octuber 1974, pp. 256-260.

8. D. B. Estreich, Ph.D., Dissertation, in progress.

9. W. R. Dawes, private communication.

$A O:$ orc: $2144: 08 / 05 / 79$ 